\title{
DESMOVILIZADOS ¿A QUÉ TIPO DE PROBLEMAS PSICOSOCIALES SE
} ENFRENTAN?

\author{
Demobilized What kind of psychosocial problems do they face?
}

Juliana Alarcón Cárdenas julianaalarcon27@gmail.com

Jeison Camilo Fonca Avila kamilofonka.24@gmail.com

Mariana de los Angeles Villarraga mavillarraga@poligran.edu.co

Institución Universitaria Politécnico Grancolombiano Psicología

Bogotá, Colombia

Resumen

El enfrentamiento que vivió Colombia por décadas ha dejado diferentes afectados, entre estos, las víctimas directas del conflicto, como también la población que decidió abandonar las armas y reinsertarse a la vida civil. Mediante este artículo de investigación cualitativa con diseño fenomenológico e investigación-acción, se pretende comprender los problemas psicosociales a los que se ven enfrentados los desmovilizados de la localidad 19, del barrio Ciudad Bolívar, BogotáColombia.

Para explorar las experiencias de vida y poder plantear estrategias de apoyo, se seleccionó una muestra de 20 individuos de tipo voluntarios, utilizando como técnicas de recolección de información entrevistas semiestructuradas y grupos focales.

Dentro de la revisión literaria, se encontró que, el mayor desafío de la Agencia Colombiana para la reintegración es ayudar a los desmovilizados a entrar al mercado laboral, ya que muchos de ellos, son personas sin estudios y en un gran porcentaje son analfabetas.

Palabras clave:

Desmovilizados, reinserción civil, conflicto armado, problemas psicosociales. 


\section{INTRODUCCIÓN}

Colombia es un país que vivió un conflicto interno armado de más de 5 décadas y que hoy, luego del proceso de acuerdo de terminación de dicho conflicto, empieza un proceso de transición hacia lo que los expertos denominan "pos-acuerdo". En ese marco, son muchas las personas que estuvieron alzadas en armas y que ahora tienen el reto de vivir en comunidades, resolviendo sus problemas sin necesidad de acudir a la violencia. Reconocer, analizar, y entrar a preguntarle a estos actores cuáles consideran que son los desafíos en la resolución de problemas que tienen, es el propósito de este trabajo.

Para entender el impacto de la reinserción de los desmovilizados, esta investigación consta de tres partes; en la primera parte, se encontrará un breve contexto histórico sobre la independencia de América Latina; como segundo apartado, se hará hincapié en cómo surgió el conflicto armado en Colombia y los intentos que hubo para finalizarlo; y para terminar el hilo teórico, como tercer apartado, se definirán los problemas psicosociales y a cuales de ellos se ven enfrentados los individuos de este estudio.

\section{Independencia de América Latina}

Luego de la colonización por parte de los Españoles a América Latina en 1492, se evidencia por primera vez las relaciones de poder, los colonizadores formaron una jerarquía piramidal donde se situaban a sí mismos en la cima, todo el que pensara diferente fue sometido de forma violenta a acatar órdenes, el tener percepciones distintas hizo que se presentara una discrepancia en la construcción de la realidad, la desigualdad era ostensible, tras comenzar las revoluciones hispanoamericanas, la lucha por intereses políticos, económicos, sociales y la idea de libertad, lleva a la independencia por parte de los criollos, mestizos, indígenas, negros, mulatos y zambos.

Sin embargo, según Salomón Kalmanovitz (2008), la independencia deja en evidencia la inexperiencia burocrática, se presentaban problemas que no podían solucionar por su inestabilidad política.

Ese conflicto de intereses políticos llevó Colombia a mediados de los sesenta ha enfrentar una guerra bipartidista entre Liberales y Conservadores, quienes eran los únicos dos partidos que tenían participación en el poder, esto desató la mayor disidencia que vivió el país por más de cinco décadas.

\section{Surgimiento del Conflicto Armado}

En América Latina, la violencia siempre ha sido utilizada como mecanismo de persuasión, sin embargo, en Colombia, tras el asesinato del líder social Jorge Eliécer Gaitán en 1948, se vivió la mayor alza en las tasas de homicidio.

Como ya se mencionó de forma somera en el primer apartado, la lucha entre liberales y conservadores generó gran confrontación social, el país se dividía en dos, por un lado, estaban los que apoyaron las guerrillas liberales y por el otro, los que estaban a favor de las bandas conservadoras. En un intento de finalizar este conflicto entre partidos para unificar el país, se construye el Frente Nacional en 1957.

A pesar de que el gobierno trató de acabar con el conflicto que se presentaba en el país, surge el movimiento guerrillero que ahora conocemos como Fuerzas Armadas Revolucionarias Colombianas (FARC), conformado por campesinos a quienes les habían quitado de forma violenta sus tierras e individuos del partido comunista que siempre habían sido excluidos por la política(Calle-Álvarez, CalleÁlvarez, \& Gómez-Sierra, 2020; Moreno-López, Sánchez-Torres, Pérez-Raigoso, \& Alfonso-Solano, 2020; Eduardo Norman-Acevedo \& Daza-Orozco, 2020). 
En principio, este movimiento fue creado con la finalidad de hacer escuchar minorías que querían defender su territorio, pero para la década de los ochenta, las FARC empieza a hacer alianza con narcotraficantes, hecho que conlleva al surgimiento de grupos de Autodefensa quienes son apoyados por las grandes élites que estaban interesadas en quedarse con dichos territorios, esto dio pie a una avalancha de asesinatos(Flórez-Romero Diana Paola; Castillo-Triana, Nicolás; Contreras-Ruiz, Paula Katerine; Henao-Floréz, Andrés; Ramírez-Toro, Diana Milena; Zárate-Pinto, Germán Camilo; GuzmánRodríguez, Rosa Julia; Anzelín-Zuluaga, Ingrid Carolina; Ruiz-He, Nicolás, 2020).

"En este periodo la tasa de homicidios se triplicó al pasar de 25 homicidios por cada 100.000 habitantes en 1974 a 79 en 1991" (bello,C; 2008)

Luego del fraude electoral que ocurrió el 19 de abril de 1970 donde fue nombrado presidente Andrés Pastrana por encima del candidato Gustavo Rojas, surge el movimiento M-19, por su ideal nacionalista acontecieron varios golpes de opinión, lo que hizo que grupos guerrilleros como las FARC tomará más fuerza.

Las causales de este conflicto son variadas, se evidencia entre ellas algunas económicas, políticas e incluso sociales, muchas regiones del país han sido segregadas a lo largo de toda la historia, esa desigualdad e injusticia pudo ser un factor desencadenante. Según Lilian Yaffe (2011), este tipo de irregularidades socioeconómicas están vinculadas de forma muy estrecha con el conflicto armado del país (Benítez, Quintero, Márquez, \& Ayala, 2015; García, 2015; Hernández, 2016; Mendoza, 2016; Urquijo, Antilef, \& Ramírez, 2016).

Colombia estuvo muy cerca de abrir mesas de discusión para decir adiós a las armas y finalizar el conflicto, el primer acercamiento a un proceso de paz se dio bajo el gobierno de Belisario Betancur entre (1982-1986), estableció que Colombia necesitaba mejorar su democracia y empezar a ver a la oposición como actor político, se acuerda un cese al fuego, sin embargo, más adelante, con el gobierno de Virgilio Barco entre (1986-1990) se rompe este acuerdo, mostró más interés por legitimar el Estado que por finalizar el conflicto, los procesos de paz permanecieron en inercia durante tres años, luego tras el gobierno de César Gaviria entre (1990-1994), se decide separar el conflicto armado de los interés políticos establecidos en la creación de la guerrilla, a pesar de ello, y con la muerte de líderes sociales como Luis Carlos Galán, Carlos Pizarro y muchos otros, se presentó la mayor crisis política de los noventa, esto salió menoscabando los acuerdos de paz. Llega al poder Ernesto Samper entre (19941998) quien retoma la postura de Betancur y tiene en cuenta a la guerrilla como actor político con quien se puede generar acuerdos.

Cuando es elegido presidente de la república Andrés Pastrana entre (1998-2002), un gran avance para los procesos de paz bajo su cargo fue crear "la zona desmilitarizada", denominada "El Caguán" pero las negociaciones no tuvieron mayor éxito, sobre Pastrana existía una presión social por parte del pueblo, quien lo tildaban de permisivo. Finalmente, antes terminar su periodo al mando, expresa que la solución del conflicto es, atacar a las personas armadas con más armas. Y en ese momento, llega al poder Álvaro Uribe Vélez, (2002-2010) teniendo como premisa, "Mano firme y corazón gigante”.

Su mandato fue muy cuestionado por el reforzamiento paramilitar, quienes más adelante apoyaron su reelección; en primera instancia, se creyó que Uribe Vélez negociaría con las FARC para la liberación de varios secuestrados, pero tras el ataque de un carro bomba a la Escuela Superior de la Guerra, decide retroceder, aunque no tenía pruebas claras, decide culpar a las FARC. Muchas familias de los secuestrados hicieron presión para que se llevarán a cabo las negociaciones, finalmente, Uribe permite que se realice la Operación Jaque, pero mantiene su postura de mano firme, entre (2010-2018) llega a la presidencia Juan Manuel Santos, bajo su gobierno se suscribieron los acuerdos, mientras las partes negociaban un acuerdo bilateral, se acordó un cese de armas y levantar las órdenes de captura; en el 
2016, se firma el "Acuerdo General para la terminación del conflicto y la construcción de una paz estable y duradera".

Luego de llevarse a cabo la firma de los acuerdos de paz, muchos desmovilizados volvieron a unirse a la vida en comunidad, tomando la decisión de afrontar todos los desafíos que esto pudiera conllevar. Dentro de los acuerdos se tienen en cuenta cinco postulados importantes, el primero es sobre la política de desarrollo agrario; el segundo, es darle la posibilidad a las FARC de ser partícipe de la política del país; el tercero, como ya se mencionó antes, es el cese de armas; el cuarto, es el cultivo y producción de drogas; y por último, la reparación a las víctimas.

El Estado colombiano, que consta de 49,07 millones de personas, 50 mil víctimas piden reparación en los procesos de justicia y paz contra los paramilitares, en promedio 602 personas son desplazadas diariamente y solo el $45 \%$ son aceptadas en el registro oficial; en los reportes del año 2006 de la oficina del Alto comisionado para la Paz y el programa de Atención Humanitaria al Desmovilizado se dijo que 3.295 paramilitares y 6.340 guerrilleros se habían desmovilizado de manera individual, y que en los procesos de desmovilización colectiva de los grupos de paramilitares, se habían obtenido por encima de 30.000 combatientes (Medios para la paz, 2007).

Bajo este contexto de guerra, la realidad se configura de una manera donde el conflicto es el eje central, tanto de la "estructuración objetiva del ordenamiento social, como en la modelación subjetiva del marco de referencia de los grupos y personas" (Martin-Baró, 2003, pág. 312). Sin embargo, el conflicto armado colombiano tiene múltiples actores, lo que genera la irrigación a través de todo el territorio nacional de manera muy diversa y produciendo diferencias en la forma como se ordena todo el país.

A Bogotá, llegan personas provenientes de diferentes zonas, víctimas en diversas situaciones y de diferentes grupos armados, conllevando un encuentro de estos en ciertas zonas de la ciudad. Caracterizar el conflicto, resulta ser un elemento definitivo del análisis realizado al proceso de reintegración comunitaria. Como decía Gustavo Duncan (2006) "la forma como los ejércitos privados dominan a la sociedad, las características de la producción de las riquezas y las relaciones entre el poder real y los individuos que conforman las comunidades políticas determinan una de las características fundamentales del orden social".

No obstante, debido a que las dinámicas del conflicto armado en Colombia no son estáticas, resulta muy difícil tratar de esclarecer las particularidades del mismo en las zonas, puesto que, así como los grupos armados se van movilizando de un lado a otro, igual lo hace la población civil, obteniendo como resultado sociedades compuestas por individuos que han vivenciado el conflicto desde distintas perspectivas y en distintas regiones, generando convivencias, interacciones y afrontamiento de nuevas formas de sobrevivir.

\section{Problemas psicosociales}

Con la aparición de las Fuerzas Armadas Revolucionarias de Colombia (FARC), surgió una ola de violencia que dejó muchas víctimas con estrés postraumático, el bienestar psicológico de la víctima como del victimario, puede verse afectado bajo situaciones estresantes "el caos y la violencia incrementa los riesgos psicológicos y de hecho el conflicto armado no sólo generan discapacidades fisiológicas, sino que también dejan una marca individual y colectiva" (Rodríguez, J; Torre, A; Miranda, C; 2002)

Situaciones como sacar a compañeros heridos en combates por minas o el ser obligado a generar algún tipo de violencia en personas secuestradas o población civil, son factores que afectaron la salud mental de los desmovilizados. 
Según la Agencia Colombiana para la reintegración, 49.000 de los 59.000 desmovilizados se han acogido a las políticas de integración, sin embargo, ha sido muy difícil entrar en el mercado laboral, ya que aproximadamente el $75 \%$ de ellos son analfabetas.

Lo que ha llevado a que muchos de ellos consigan trabajos informales, trabajando bastantes horas al día, sin una remuneración salarial justa.

\section{Método}

\section{Tipo de Investigación}

Existen varios tipos de investigación científica dependiendo del método y de los fines que se persiguen. La investigación, de acuerdo con Hernández, Fernández y Baptista (2014) y Daza-Orozco (2019) la definen como: "un conjunto de procesos sistemáticos, críticos y empíricos que se aplican al estudio de un fenómeno o problema" (p. 4).

Por ende, el tipo de investigación que favorece y posteriormente se utiliza para el presente trabajo es cualitativa, de acuerdo con Hernández, et al. (2014) afirman que: "la investigación cualitativa utiliza la recolección y análisis de los datos para afinar las preguntas de investigación o revelar nuevas interrogantes en el proceso de interpretación" (p.7), además, se basa en métodos de recolección de datos no estandarizados, ni predeterminados completamente. Tal recolección, consiste en obtener las perspectivas y puntos de vista de los participantes (sus emociones, prioridades, experiencias, significados y otros aspectos más bien subjetivos). También resultan de interés las interacciones entre individuos, grupos y colectividades. El investigador hace preguntas más abiertas, recaba datos expresados a través del lenguaje escrito, verbal y no verbal, así como visual, los cuales describe, analiza y convierte en temas que vincula, y reconoce sus tendencias personales. Debido a ello, la preocupación directa del investigador se concentra en las vivencias de los participantes tal como fueron (o son) sentidas y experimentada. (Hernández, et al., 2014, p. 9).

\section{Diseño de Investigación}

El abordaje general que se utiliza en el presente proceso de investigación es de tipo fenomenológico y diseño Investigación-acción (IAP). El primero, plantea como propósito principal "explorar, describir y comprender las experiencias de las personas con respecto a un fenómeno y descubrir los elementos en común de tales vivencias" (Hernández, Fernández y Baptista, 2014, p. 493). Posteriormente, en este caso, aplica un enfoque fenomenológico empírico, en donde se enfoca menos en la interpretación del investigador y más en describir las experiencias de los participantes (E. Norman-Acevedo, 2018).

Para Creswell, 2013 y Wertz et al. 2011 (como se citó en Hernández, et al., 2014) en los procedimientos básicos incluyen: a) determinar y definir el problema o fenómeno a estudiar, b) recopilar los datos sobre las experiencias de diversos participantes con respecto al mismo, c) analizar los comportamientos y narrativas personales para tener un panorama general de las experiencias, d) identificar las unidades de significado y generar categorías, temas y patrones, detectando citas o unidades clave; e) elaborar una descripción genérica de las experiencias y su estructura (condiciones en las cuales ocurrieron, 3 las narrativas?) y las diferentes apreciaciones, valoraciones u opiniones sobre éste (categorías y elementos distintos: ¿en qué cuestiones difieren las múltiples narrativas de las experiencias?) (Hernández, et al., 2014, p. 494). 
Por su parte, el segundo diseño de esta investigación es de tipo Investigación-Acción Participativa o Cooperativa, que de acuerdo con como lo afirman Hernández, Fernández y Baptista (2014): “En ésta los participantes pueden fungir como coinvestigadores ya que necesitan interactuar de manera constante con los datos. Del planteamiento a la elaboración del reporte" (p. 501); Asimismo, en estos diseños también se resuelve una problemática o se implementan cambios, pero en ello intervienen de manera aún más colaborativa y democrática uno o varios investigadores y participantes o miembros de la comunidad involucrada, por lo que, en efecto, la problemática es identificada en conjunto por la comunidad y los investigadores. Se considera a los miembros de la comunidad como expertos en la misma, por tal motivo sus "voces" resultan esenciales para el planteamiento y las soluciones, es por ello, que, durante todo el proceso, los miembros de la comunidad son considerados como "socios" y altamente valuados por sus perspectivas únicas y la información que proporcionan (Hernández, et al., 2014, p. 501).

\section{Participantes y tipo de muestra}

La muestra fue de tipo voluntaria, explicada por Hernández, et al., (2014), como: "En estos casos, la elección de los participantes depende de circunstancias muy variadas. A esta clase de muestra también se le puede llamar autoseleccionada, ya que las personas se proponen como participantes en el estudio o responden a una invitación" (p. 420).

Se contó con los relatos de 20 personas, pertenecientes a 4 grupos poblacionales en particular, víctimas, desmovilizados, comunidad de acogida y líderes sociales, de diferentes edades, pertenecientes a la localidad $\mathrm{N}^{\circ} 19$, Ciudad Bolívar de Bogotá D.C, en donde 15 de ellas fueron convocados a colaborar voluntariamente en los grupos focales. Los participantes fueron reunidos en 3 oportunidades a lo largo del proyecto y gracias a sus opiniones, se lograron obtener diferentes perspectivas para dar una culminación exitosa a los objetivos del proyecto planteados inicialmente.

\section{Instrumentos}

Se tienen en cuenta tres tipos de instrumentos, la entrevista semi-estructurada, el grupo focal y la cartografía social, a continuación, se expondrán cada uno de los mencionados,

\section{Entrevista Semi-estructurada}

Para la recolección y posterior análisis de los datos de los relatos planteados por los participantes, el instrumento principal usado dentro de este proyecto es la entrevista cualitativa. Esta se define como un espacio de reunión entre dos o más personas, donde se busca socializar y crear un espacio para conversar el cual está compuesto por el entrevistador y el entrevistado o entrevistados. Una de las principales características de la entrevista cualitativa está en que esta suele ser más íntima por lo que busca generar esos espacios abiertos de reflexión a lo largo de su proceso. La entrevista consta de tres tipos, estructurada, semiestructurada y abierta (Hernández, Fernández y Baptista, 2014). El presente Proyecto de Investigación Formativa (PIF) ha decidido centrarse en la entrevista semiestructurada debido a que esta se compone de un guion previo que va acorde a la temática a tratar dentro de la intervención, pero nos da la libertad de poder profundizar en muchos más temas

\section{Grupo focal}

En esta investigación se usó la técnica de Grupo Focal, la cual la define Korman, (1986) como: "una reunión de un grupo de individuos seleccionados por los investigadores para discutir y elaborar, desde la experiencia personal, una temática o hecho social que es objeto de investigación” (pp. 1)

\section{Cartografía Social}

Vélez, Rativa, Valera (2012), "metodología participativa y colaborativa de investigación que invita a la reflexión, organización y acción alrededor de un espacio físico y social específico" (pp. 4), este instrumento ayuda a visualizar puntos estratégicos dentro de la comunidad. 
Tabla 1. Instrumentos de recolección de datos

\section{Resultados}

La investigación se encuentra aún en desarrollo, razón por la que aún no se cuenta con resultados definitivos.

\section{Discusión y Conclusión}

Se evidencia una gran preocupación por parte de los desmovilizados, donde el factor económico y el nivel académico se convierten en obstáculos para poder cumplir los requisitos mínimos al momento de solicitar un empleo.

Los estigmas de la sociedad colombiana en general tienden a imponer una barrera por parte de las empresas del sector privado en incluir a personas desmovilizadas dentro de su grupo de trabajo, y como lo menciona Turriago, D (2016), en ellos se instaura una herencia emocional producto del malestar de la violencia, la cual debe tomarse en cuenta en los procesos actuales de reparación, "la sociedad democrática deberá enfrentar la posible herencia del miedo que se recibe de estas dictaduras militares, si no se quiere vivir permanentemente amarrado a traumas y fantasmas que de una u otra manera se harán presentes"

Los desmovilizados y las comunidades de acogida se enfrentan a múltiples problemas cotidianamente, que los desbordan pero que siempre que cuenten con grupos vecinales que les apoyen podrán salir adelante. Sin embargo, de esta forma, la superación del malestar vigente cuando se ha vivido en la guerra no es suficiente y no se logrará alcanzar a menos que se reconozcan las pérdidas y ganancias, las emociones y fortalezas que se configuraron al interior del conflicto, sin olvidar o negar la vivencia, como lo menciona Baró, I. (1990) “una reparación real solamente será posible reconociendo lo que se destruyó, integrando lo que se perdió, construyendo un futuro con fuerza y debilidad; con alegría y tristeza; integrando y construyendo la historia, sin olvidarla ni negarla.

\section{Referencias bibliográficas}

Benítez, P., Quintero, M., Márquez, K., \& Ayala, A. O. (2015). Beneficios y garantías de los miembros de la fuerza pública en marcos de justicia transicional en perspectiva comparada: lecciones para el caso colombiano. Panorama, 9(16), 105-116. https://doi.org/10.15765/pnrm.vgi16.630

Baró, I. (1990a). Guerra y Salud Mental. En I. Martín-Baró (Ed.) Psicología Social de la Guerra: trauma y terapia. San Salvador: UCA editores, pp. 23-40.

Bello Montes, Catalina. (2008). La violencia en Colombia: Análisis histórico del homicidio en la segunda mitad del Siglo XX. Revista Criminalidad, 50(1), 73-84. Recuperado de: http://www.scielo.org.co/scielo.php?script=sci_arttext\&pid=S1794$31082008000100005 \& \operatorname{lng}=$ en\&tlng=es.

Calle-Álvarez, G., Calle-Álvarez, G. Y., \& Gómez-Sierra, M. I. (2020). EL COMPORTAMIENTO LECTOR EN TEXTOS MULTIMODALES DIGITALES EN LA BÁSICA PRIMARIA. Panorama, 14(27), 14-34. https://doi.org/10.15765/pnrm.v14i27.1518

Chernick, M. Introducción. Aprender del pasado: Breve historia de los procesos de paz en Colombia (1982-1996). Recuperado de: https://revistas.uniandes.edu.co/doi/pdf/10.7440/colombiaint36.1996.02

Contreras, M.(2003) El conflicto armado en Colombia Revista de Derecho, núm. 19, junio, 2003, pp. 119-125 Universidad del Norte Barranquilla, Colombia. Recuperado de: https://www.redalyc.org/pdf/851/85101907.pdf

Daza-Orozco, CE. (2019). Iniciación científica: conceptualización, metodologías y buenas prácticas. Bogotá: Sociedad Colombiana de Investigación e Innovación Formativa - SCOIF. Retrieved from http://www.scoif.com/iniciacioncientifica/

Duncan, G. (2006). Libro: Los señores de la guerra. Bogotá

Flórez-Romero Diana Paola; Castillo-Triana, Nicolás; Contreras-Ruiz, Paula Katerine; Henao-Floréz, Andrés; Ramírez-Toro, Diana Milena; Zárate-Pinto, Germán Camilo; Guzmán-Rodríguez, Rosa Julia; Anzelín-Zuluaga, Ingrid Carolina; Ruiz-He, Nicolás, R. G.-M. (2020). Fundamentos para la cualificación del talento humano: primera infancia y conflicto en Colombia (1st ed.). Bogotá: Institución Universitaria Politécnico Grancolombiano.

García, V. S. (2015). La gestión local como propuesta para la solución de problemas comunes de urbanismo en América Latina. Panorama, 9(16), 92-103. https://doi.org/10.15765/pnrm.vgi16.633 
Hernández, Y. M. (2016). La Unión Patriótica: memorias para la paz y la democracia. Panorama, 10(18), 27-38. https://doi.org/10.15765/pnrm.v10i18.822

Hernández, Fernández y Baptista (2014). Metodología de la investigación, sexta edición. Disponible en: https://www.esup.edu.pe/descargas/dep_investigacion/Metodologia\%20de\%20la\%2oinvestigación\%205ta\%20Edición.pd $f$

Kalmanovitz, S (2008) Consecuencias económicas de la independencia en Colombia.

Korman, H. (1986). The Focus Group Sensing. Dept. Of Sociology, SUNY at Stony Brook. New York.

López, M (2004). Enciclopedia de paz y conflictos. Tomo I. Colección Eirene. Instituto de la paz y los conflictos. Granada: Universidad de Granada.

Mendoza, J. A. G. (2016). Empresa privada: principal socio en el posconflicto y la construcción de la paz. Panorama, 10(18), 8492. https://doi.org/10.15765/pnrm.v10i18.823

Moreno-López, N. M., Sánchez-Torres, A. I., Pérez-Raigoso, A. D. P., \& Alfonso-Solano, J. N. (2020). TRADICIÓN ORAL Y TRANSMISIÓN DE SABERES ANCESTRALES DESDE LAS INFANCIAS. Panorama, 14(26), 184-194. https://doi.org/10.15765/pnrm.v14i26.1489

Moreno, A. (2006). Transformaciones internas de las FARC a partir de los cambios políticos por los que atraviesa el estado colombiano.

Papel Politico, 11(2), 595-646. Retrieved October 30, 2019, from http://www.scielo.org.co/scielo.php?script=sci_arttext\&pid=S0122-44092006000200005\&lng=en\&tlng=pt.

Norman-Acevedo, E. (2018). Rompiendo Barreras, 10 Āños de la Educación Virtual en el Politécnico Grancolombiano. Bogotá. Politécnico Grancolombiano. Retrieved from http://alejandria.poligran.edu.co/handle/10823/1146

Norman-Acevedo, E, \& Daza-Orozco, CE. (2020). Construcción de contenidos para la enseñanza virtual: retos coyunturales en el confinamiento. Panorama, 14(27), 5-13. https://doi.org/10.15765/pnrm.v14i27.1517

Rodríguez, J. Torre, A. Miranda, C. (2002) La salud mental en situaciones de conflicto armado Biomédica, vol. 22, núm. Su2, diciembre, 2002, pp. 337-346 Instituto Nacional de Salud Bogotá, Colombia. Recuperado de https://www.redalyc.org/pdf/843/84309603.pdf

Stromquist, N. (2006). Una cartografía social del género en educación. Educ. Soc., 27(95), pp. 361-383 Recuperado de: http://www.scielo.br/pdf/\%oD/es/v27n95/a03v2795.pdf

Turriago, D. (2016). Los procesos de paz en Colombia, camino ia la reconciliación? Actualidades Pedagógicas, (68), 159-178. Recuperado de: :https://doi.org/10.19052/ap.3827

Urquijo, A. P. L., Antilef, J. del C. C., \& Ramírez, G. R. (2016). Desarrollo del pensamiento lógico basado en resolución de problemas en niños de 4 a 5 años. Panorama, 10(19), 98-107. https://doi.org/10.15765/pnrm.v10i19.831

Velez, T. Rátiva, G. Varela, C. (2012). Cartografía social como metodología participativa y colaborativa de investigación en el territorio afrodescendiente de la cuenca alta del río cauca. Cuadernos de Geografía: Revista Colombiana de Geografía; 21(2), pp. (59 - 73) Recuperado de: http://bdigital.unal.edu.co/27871/1/25774-121714-1-PB.pdf 